\title{
Audit on implementation of neonatal pulse-oximetry screening for critical congenital heart disease at neonatal units of District General Hospital - Kalutara
}

\author{
Mayoorathy $V^{1}$, Dias $S^{1}$ \\ ${ }^{1}$ General Hospital Kalutara. \\ Corresponded Author: Mayoorathy, V. \\ Email: s.mayoorathy@gmail.com iD https://orcid.org/0000-0002-3319-0221
}

\begin{abstract}
Pulse-oximetry screening was introduced in Sri Lanka in 2015, as a universal newborn screening for Critical Congenital Heart Diseases (CCHD), that are undetectable by clinical examination. The objective was to assess the implementation of standard protocol in CCHD screening. A pretested interviewer-administrated questionnaire was used to collect data while directly observing the procedure. Standard recommendations of the American Academy of Paediatrics were used. Screening was done in 133 (76\%). 70\% were screened after 24 hours of birth. Non- adjustable adult probe was used and it was not applied properly in all cases. Saturations were checked in two limbs in $85 \%$ and four limbs in rest.Suspicious result (19) only interpreted by a medical officer without standard interpretations. Despite of the high screening rate, standard recommendations were not followed in most.
\end{abstract}

(Key Words: Pulse oximetry, Congenital heart disease, Screening)

\section{Introduction}

The incidence of moderate to severe congenital heart disease is estimated to be $6 / 1000$ live birth with duct dependent critical congenital heart disease (CCHD) being present in 2-3 /1000 live births. (1) Pulse oximetry can assist in detecting critical congenital heart disease that are not detected clinically or by antenatal anomaly scans. (2) This audit was done to assess the compliance with the recommended protocols of pulse oximetry screening test by American Heart Association.

\section{Methods and materials}

Randomly selected 50 neonates were directly observed while undergoing the screening procedure at postnatal wards of District General Hospital
Kalutara during the month of February 2016. A pre - tested, Investigator- administrated questionnaire was used to collect the data.

Recommendations given by the American Academy of Paediatrics were used as standard to evaluate the technique and to assess the interpretation of test.(3)

\section{Results}

Total number of births were 176 and screening was done in $133(76 \%) .70 \%$ of the babies were screened after 24 hours of birth while rest of them screened between 12-24hours.

Almost all were used with adult probe for screening. Probe was applied to the finger than the fleshy part of the palm and sole in all cases as the probe was non-adjustable. Position of the emitter and photo detector were maintained in all cases as the probe was fixed one. $15 \%(n=6)$ probes were held by hands.

All 4 limb saturations were checked in neonates with murmur $(\mathrm{n}=12)$ was $30 \%$. All others were checked with right hand and one of the feet. Not each and every screening test was interpreted. Suspicious results were seen by a medical officer $(n=19)$

Only one child had positive test during the study period and referred to LRH for 2DEcho that showed obstructed TAPVD. The Baby underwent surgery on day 2.

\section{Discussion}

Screening has reached over $80 \%$ coverage and this shows the feasibility of the method and clearly dismisses concerns that this method only works in large research settings with dedicated staff and funding. It should be highlighted that even persistent $\mathrm{SpO} 2$ of $>95 \%$ in one limb is inadequate to exempt from routine screening as the difference 
of $\mathrm{SpO} 2$ between the pre ductal and post ductal is more sensitive

Checking all four limb saturation has no additional benefits in detecting the congenital heart disease and it may further complicate the process of interpretation of the test.

It is recommended that screening should not be undertaken until 24 hours of life or as late as possible if early discharge is planned to reduce the number of false - positive results. (3)

\section{Suggestion}

To improve quality in screening for CCHD, providing neonatal pulse oximetry probes and proper training of the staff on placement of probe and screening technique is paramount.

We suggest displaying the printed version of the algorithm in the screening place and training the personnel involving interpretation of the test will increase the convenience as well as the effectiveness of the screening.

\section{References:}

1. Hoffman JIE, Kaplan S. The incidence of congenital heart disease. J Am Coll Cardiol 2002: 39: 1890-1900

2. Granelli A, Alf Meberg, Ojala T, Steensberg J, Oskarsson G, Mellander M.Nordic pulse oximetry screening - implementation status and proposal for uniform guidelines. Acta Pædiatrica. 2014; 103;1136-1142

3. Endorsement of Health and Human Services-Recommendation for Pulse Oximetry Screening for Critical Congenital Heart Disease. American Academy of Paediatrics. PEDIATRICS. January 2012;129(1) 